\title{
Critical slowing down in an optical bistable model with a Kerr-nonlinear blackbody
} reservoir

\section{Y. A. Sharaby ${ }^{a^{*}}$, S. S. Hassan ${ }^{\text {b }}$, S. Lynch ${ }^{\mathrm{c}}$}

${ }^{a}$ Suez University, Faculty of Sciences, Physics Department, Suez, Egypt.

${ }^{\mathrm{b}}$ University of Bahrain, College of Science, Department of Mathematics, P.O. Box 32038, Bahrain.

${ }^{c}$ School of Computing, Mathematics and Digital Technology, Manchester Metropolitan University, Manchester M1 5GD, United Kingdom.

\section{Keywords:}

Optical bistability - critical slowing down - Kerr nonlinear blackbody radiation - switching time.

E-mail address: Yasser_sharaby@hotmail.com (Y. A. Sharaby) shoukryhassan@hotmail.com (S. S. Hassan) s.lynch@mmu.ac.uk (S. Lynch)

${ }^{*}$ Corresponding author. 


\begin{abstract}
We investigate switching response for an Optical Bistable (OB) device consisting of homogeneously broadened two-level atoms in a ring cavity supported by a Kerr Nonlinear Blackbody (KNB) radiation reservoir in the high-Q cavity regime for both absorptive and dispersive cases. In the resonant case and below a transition temperature, faster switching processes for OB devices with KNB can be triggered by a small perturbation of the incident field in the vicinity of the critical transition point. The switching time increases with increasing atomic detuning parameter. A thermal switching process is obtained for a fixed incident field and is triggered by small perturbation in the relative reservoir temperature, $\mathrm{T}_{\mathrm{b}}$ say. The switching time is reduced considerably by slightly increasing the temperature $T_{b}$. Comparison with other cases of radiation reservoir is made, namely, normal vacuum, thermal field and squeezed vacuum.
\end{abstract}




\section{Introduction}

Optical bistability is vitally important in nonlinear optical phenomena, due to its potential applications in different branches of science, optical computations, optical communications and the biological and medical sciences. It has been investigated theoretically and experimentally with dissipative two-level atomic systems placed in an optical cavity [1-4]. OB systems have attractive applications in all optical switches, memories, transistors and logic circuits $[5,6]$ with normal vacuum field. These studies show that one can control the bistable threshold intensity and the hysteresis loop via many approaches, such as field-induced transparency [7] and phase fluctuations [8]. Reversed (clockwise) and butterfly (closed loop) hysteresis structures [9] were predicted for the additional first harmonic output field component outside the Rotating Wave Approximation (RWA) simultaneously with the usual bistable (anti-clockwise) hysteresis for the fundamental output field component. The first harmonic output field component outside the RWA can be further controlled to show a one- or two-way switching processes when atomic inhomogeneous broadening and transverse input field features are taken into consideration [10]. There may be applications in optical information signal processing as well as simultaneous opposite coding.

The nonlinear atomic medium in OB devices is affected by the quantum state of the radiation reservoir responsible for the atomic damping processes as follows:

(i) The thermal Field (TF) leads to the broadening of the longitudinal and transverse atomic linewidths [4].

(ii) The Squeezed Vacuum (SV) field induces narrowing in the transverse atomic linewidth associated with one quadrature component of the 
atomic polarization [11], as a result of the simultaneous creation or annihilation of photon pairs in the squeezed state.

(iii) In the $\mathrm{KNB}$ case, the natural atomic linewidth (the spontaneous emission) is suppressed as a result of the formation of photon pairs by the phonons of lattice vibrations under the condition that $T<T_{C}$, where $\mathrm{T}_{\mathrm{C}}$ is the critical transition temperature $[12,13]$.

Optical bistable systems with injected SV field [14-19], compared with the Normal Vacuum (NV) case $[3,4]$ have their advantages of achieving optical bistbility at a lower threshold value of the atomic cooperative parameter $(C<4)$, as well achieving a one - or two - phase switching processes by adjusting the relative phase of the degree of the squeezing parameters.

On the other hand, OB system of a homogeneously broadened two-level atomic medium interacting with a single mode of the ring cavity in the presence of the Kerr-nonlinear blackbody reservoir has been studied recently in both absorptive and dispersive cases [20]. It is shown that optical bistability is observed at an even lower cooperativity parameter than that in the SV case [16]. Furthermore, a temperature induced switching process at a fixed input field is predicted near resonance conditions [20]. In the $\mathrm{KNB}$, the resulting radiation is found to be a squeezed thermal state below a certain transition temperature [21]. The significant change occurring when a normal blackbody (thermal field) is replaced by a $\mathrm{KNB}$, in matter-electromagnetic field interaction, is that the usual vacuum state of the electromagnetic field is replaced by the photon pair state and in turn the infinite energy of the field vacuum is replaced by the finite energy of the photon pairs $[13,22]$.

The phenomenon addressed in this paper, namely, Critical Slowing Down (CSD), is associated with lengthening the time taken for the system to recover 
from a small perturbation or disturbance in one of its control parameters in the vicinity of a critical transition point. As a consequence, the large delay time leads to: (i) a large memory device, and (ii) a slow switching device. Earlier, within the RWA, CSD in the NV case was examined for the absorptive optical bistability [23] and later extended to the SV case [24]. A study of CSD would benefit (at some degree) the following:

(i) Enabling decay rates of the transitional transient processes to stable steady-state to be measured.

(ii) There are possible device applications in optics [25-27].

(iii) It may be possible to achieve dynamical stabilization of the system in response to perturbations or fluctuations of the system parameters near the critical points [28].

Recently, we have examined the CSD of an OB model of two-level atoms placed inside a ring cavity outside the RWA in the high- and low-Q cavity cases [29]. The faster oscillatory behavior outside the RWA induces irregular oscillations with increased atomic detuning in the lower branch of the hysteresis curve of the first harmonic output field due to interference with atomic dispersion or Rabi oscillations. Effects of atomic inhomogeneous (Lorentzian) broadening and transverse (Gaussian) field variations on the CSD in the high-Q cavity limit has been discussed in [30]. The main result in [30] in the high-Q cavity case is that, the switching time decreases with increasing the Lorentzian parameter in both absorptive and dispersive cases. In addition, the transverse field parameter increases the switching response of the optical bistable device significantly in the dispersive case with associated irregular oscillations in the lower branch.

Elsewhere [31,32], CSD was investigated for some bistable biological and environmental models. In [31] it was shown that the time-delay reduction is 
independent of the nonlinearity form and fits an inverse square root law $\beta^{-1 / 2}$, where $\beta$ is the perturbation parameter (see e.g. [25-26] and refs. therein). In [32] it was suggested that CSD could provide universal indicators of how close a complex system such as the brain, the climate, ecosystems and the financial markets, are to a threshold. CSD applied to the optical properties of atoms is covered in papers such as $[33,34]$.

The aim of the present work is to study the switching response of an OB system of 2-level atoms in the presence of KNB reservoir in the vicinity of critical transition point and compare it with previously studied radiation reservoir cases, namely, the NV, TF and SV reservoirs. This is achieved by showing the effect of perturbations of the incident field near a transition point. Furthermore, we investigate the thermal switching effects by perturbing the relative temperature $\mathrm{T}_{\mathrm{b}}$ in the vicinity of critical value of $T_{C}$.

The paper is organized as follows: A review of our model is presented in Sec. II. Both incident field and thermal switching responses in the OB model in the highQ cavity limit is examined in Sec. III. A Summary is given in Sec. IV.

\section{Model review}

Consider a single mode ring cavity containing a homogeneously broadened twolevel atomic medium in contact with a thermal reservoir of temperature $T_{B}$ and of transition frequency $\omega_{0}$ and interacting with an electromagnetic field of frequency $\omega_{\mathrm{L}}$. The coherent interaction between atoms and field that propagates along the longitudinal axis induces macroscopic polarization and changes in the level population of the atomic system. The c-number model Maxwell-Bloch 
equations in the plane wave, rotating wave and mean field approximations are given by [20]:

$$
\begin{aligned}
& \frac{\mathrm{dx}}{\mathrm{dt}}=\kappa\left[\mathrm{Y}-(1+\mathrm{i} \theta) \mathrm{x}+2 \sqrt{2} \mathrm{Cr}_{-}\right], \\
& \frac{\partial}{\partial t} r_{-}=-\frac{\gamma}{2}\left(1+2 n_{1}+i \delta\right) r_{-}+\frac{\gamma}{\sqrt{2}} r_{3} x=\left(\frac{\partial}{\partial t} r_{+}\right)^{*}, \\
& \frac{\partial}{\partial \mathrm{t}} \mathrm{r}_{3}=-\frac{\gamma}{2}\left(1+2\left(1+2 \mathrm{n}_{1}\right) \mathrm{r}_{3}\right)-\frac{\gamma}{2 \sqrt{2}}\left(\mathrm{r}_{+} \mathrm{x}+\mathrm{r}_{-} \mathrm{x}^{*}\right) .
\end{aligned}
$$

Here, $\mathrm{n}_{1}=1 /\left(\mathrm{e}^{\hbar_{\omega} / \mathrm{k}_{\mathrm{B}} \mathrm{T}_{\mathrm{B}}}-1\right)$ is the average photon number of the heat bath (thermal reservoir) maintained at a fixed temperature $T_{B}$, with central frequency $\omega$, Boltzmann constant $\mathrm{k}_{\mathrm{B}}$ and $\delta=2\left(\omega_{\mathrm{L}}-\omega_{\mathrm{o}}\right) / \gamma$ is the normalised atomic detuning where, $\gamma$ is the A-coefficient. The notations in Eq. (1) are as follows: $r_{ \pm}$are the mean values of the quadrature atomic polarization components, $r_{3}$ is the mean value of the atomic inversion. The quantities $\mathrm{x}$ and $Y$ are the normalized output and input amplitude fields, respectively, $\theta=\left(\omega_{c}-\omega_{L}\right) / \kappa$ is the normalized cavity detuning with $\omega_{c}=$ cavity mode frequency, and $\kappa=$ cavity decay constant and $C=g^{2} /(\gamma \kappa)$ is the cooperative parameter, where $g$ is the coupling between the cavity field and the atoms.

In the steady-state, equations (1) yield the well-known input-output field steady state equation [4]

$$
\mathrm{Y}=\mathrm{x}\left[1+\frac{2 \mathrm{C}}{\left(1+2 \mathrm{n}_{1}\right)^{2}+\delta^{2}+|\mathrm{x}|^{2}}+\mathrm{i}\left(\theta-\frac{2 \mathrm{C} \delta}{\left(1+2 \mathrm{n}_{1}\right)\left(\left(1+2 \mathrm{n}_{1}\right)^{2}+\delta^{2}+|\mathrm{x}|^{2}\right)}\right)\right] .
$$

In the case of KNB reservoir [13, 24], the radiation is in a squeezed thermal state below a transition temperature $T_{c}$ (dependent on the Kerr nonlinear crystal), 
which results in atomic spontaneous emission suppression due to formation of photon pairs via lattice vibrations. Above the temperature $T_{c}$, the KNB behaves like a normal blackbody (ordinary thermal radiation of temperature $\mathrm{T}_{\mathrm{B}}$ ). The formation of photon pairs is physically understood as follows [11]: if one photon (first photon) is surrounded by a cloud of lattice vibrations (phonons) then with another photon nearby this polarization cloud, it experiences a force of attraction with the first photon and a photon pair is then formed. Not all KNB photons are paired. Unpaired photons form a new kind of quasiparticles, the nonpolaritons. Hence, spontaneous emission of atomic system coupled to a KNB reservoir (i.e., a thermal reservoir with KNB medium) is modified (suppressed) as result of this photon pairing process for $T_{B}<T_{c}$. Accordingly, the decay rates ( $w_{h e n} T_{B}<T_{c}$ ) in Eq. (1) are modified as follows [13]:

$\gamma \rightarrow \Gamma_{r} \gamma \quad\left(0<\Gamma_{r}<1\right)$

where, the relative decay rate $\Gamma_{\mathrm{r}}$ is given by

$\Gamma_{\mathrm{r}}=\left(\frac{1-\Delta\left(\mathrm{T}_{\mathrm{B}}\right)}{1+\Delta\left(\mathrm{T}_{\mathrm{B}}\right)}\right)^{1 / 2} \mu$

with $\Delta\left(T_{B}\right) \neq 0$ (for $T_{B}<T_{c}$ ) is the order parameter for pairing of photons, which is a monotonically decreasing function of the temperature $\left(T_{B}\right)$ of the reservoir and vanishes at the transition temperature $T_{c}$ and yields $\Gamma_{r} / \mu$ with $\mu$ is the refractive index (dispersion free) of the medium of KNB. Note that the order parameter $\Delta\left(\mathrm{T}_{\mathrm{B}}\right)=0$ in the two cases: the normal blackbody thermal reservoir $\left(T F, T_{B} \geq T_{c}\right)$ and $N V\left(T_{B}=0\right)$ cases, where $\Gamma_{r}=1$. An approximate expression of $\Delta\left(\mathrm{T}_{\mathrm{B}}\right) \simeq \sqrt{2} \sqrt{1-\left(\mathrm{T}_{\mathrm{B}} / \mathrm{T}_{\mathrm{c}}\right)}$, which holds well near $\mathrm{T}_{\mathrm{c}}$ [32]. In OB with KNB case, the Maxwell-Bloch equations (1), after using (3), are modified to the following: 


$$
\begin{aligned}
& \frac{d x}{d t}=\kappa\left[Y-(1+i \theta) x+2 \sqrt{2} C r_{-}\right], \\
& \frac{\partial}{\partial t} r_{-}=-\frac{\gamma}{2}\left(\Gamma_{r}\left(1+2 n_{1}\right)+i \delta\right) r_{-}+\frac{\gamma}{\sqrt{2}} r_{3} x=\left(\frac{\partial}{\partial t} r_{+}\right)^{*}, \\
& \frac{\partial}{\partial t} r_{3}=-\frac{\gamma}{2}\left(\Gamma_{r}+2 \Gamma_{r}\left(1+2 n_{1}\right) r_{3}\right)-\frac{\gamma}{2 \sqrt{2}}\left[r_{+} x+r_{-} x^{*}\right] .
\end{aligned}
$$

In the steady-state, equations (5) yield to the following input-output relation [22],

$$
Y=x\left[1+\frac{2 C \Gamma_{r}}{\Gamma_{r}^{2}\left(1+2 n_{1}\right)^{2}+\delta^{2}+|x|^{2}}+i\left(\theta-\frac{2 C \delta}{\left(1+2 n_{1}\right)\left(\Gamma_{r}^{2}\left(1+2 n_{1}\right)^{2}+\delta^{2}+|x|^{2}\right)}\right)\right]
$$

Next, we investigate the switching response of the system through two processes, namely;

i. $\quad$ Field switching process, by perturbing the incident field with small perturbations in the neighberhood of the crtical (switching) point of the incident field.

ii. Thermal swiching process which is triggered by small perturbations of the KNB reservoir temperature $T_{B}$ in the neighberhood of the crtical point of the relative temperature $T_{b}=T_{B} / T_{C}$.

\section{Critical slowin down in the high-Q cavity case}

\section{(a) Field switching}

In the high-Q cavity case, the life time of photons inside the cavity $\left(\kappa^{-1}\right)$ is much greater than the atomic lifetime $\left(\gamma^{-1}\right)$ and hence the atomic variables can be eliminated adiabatically from equations (5a-c). Accordingly, equations (5) reduce to the single differential equation for the output field: 


$$
\frac{d x}{d \tau}=Y-(1+i \theta) x(\tau)-2 C x(\tau) \frac{\left(\Gamma_{r}\left(1+2 n_{1}\right)-i \delta\right)}{\left(1+2 n_{1}\right)\left(\Gamma_{r}^{2}\left(1+2 n_{1}\right)^{2}+\delta^{2}+\left|x_{o}(\tau)\right|^{2}\right)}
$$

Note, equation (7) covers the three cases of $\operatorname{NV}\left(\Gamma_{r}=1, n_{1}=0\right), \operatorname{TF}\left(\Gamma_{r}=1, n_{1} \neq 0\right)$, KNB $\left(0<\Gamma<1, n_{1} \neq 0\right)$ cases. The case for the SV is described by the following state equation [15]:

$$
\frac{d x(\tau)}{d \tau}=Y-x(\tau)-\frac{2 C\left(b_{1}-i b_{2}\right) x(\tau)}{1+\delta^{2}+b_{1}|x(\tau)|^{2}}
$$

where $\tau=\kappa \mathrm{kt}, b_{1}=1-\frac{2|M| \cos \Phi}{1+2 N}, b_{2}=\frac{\delta+2|M| \sin \Phi}{1+2 N}$ and $\Phi=\phi_{s}-2 \phi_{f}$ is the relative phase of the squeezed vacuum field with respect to the output field. The squeezed vacuum parameters: $\mathrm{N}=$ average photon number and $M=|M| e^{i \phi_{s}}=$ the degree of squeezing, are related for maximum squeezing by $|M|^{2}=N(1+N)$. Now we investigate the switching time of the OB device in the vicinity of the critical switching-on point for cases of $\mathrm{SV}\left(\Phi=\pi, n_{1}=0.158237\right), \mathrm{NV}\left(\Gamma_{r}=1, n_{1}=0\right)$, $\operatorname{KNB}\left(\Gamma_{r}=1 / 2, n_{1}=0.158237\right), \operatorname{TF}\left(\Gamma_{r}=1, n_{1}=0.158237\right)$ by solving equations $(7,8)$ independently, with a linear perturbation of the incident field $\left(\mathrm{Y}_{\mathrm{c}}+\beta\right) ; 0<\beta<1$, evaluated at the critical points from eq. (6) and (8). Here, equations $(7,8)$ are numerically integrated using Mathematica ${ }^{\circledR}[36]$ with steady-state initial conditions obtained by $(6,8)$.

In the absorptive case, Fig. (1), the computational results show that for fixed field perturbation $\beta=0.03, C=20$, the delay time is much reduced for the KNB case compared with other cases of SV, NV and TF by factors of $0.133,0.0555$ and $4 \times 10^{-10}$,respectively. Further, in the KNB case, the switching response of the optical bistable device is reduced by increasing $\beta$ (Fig. 2). In the dispersive case, Fig. 3, for fixed $\beta=0.04, C=20$ the increase in the atomic detuning parameter $\delta$ leads to an increase in the delay time. 


\section{(b) Thermal switching}

In this subsection, we investigate the thermal switching of the OB device with KNB by perturbing the relative temperature $T_{b}$ around its critical value $\left(T_{b c}\right)$, for fixed incident field value $Y$ and different values of the thermal perturbations $\beta_{\mathrm{T}}$ (Fig. 4). The relation between the output field $|x|$ and temperature $T_{b}[20]$ is shown in the inset of Fig. (4), for fixed $Y=80, C=40, \Gamma_{r}=\delta=\theta=1 / 12$. Thermal switching process for the OB device with KNB occurs by solving equation (7) numerically at fixed $\mathrm{Y}=80$ and replacing $\mathrm{T}_{\mathrm{b}}$ by $\mathrm{T}_{\mathrm{bc}}+\beta_{\mathrm{T}}\left(\mathrm{T}_{\mathrm{bc}}=0.543\right.$ which is obtained from the inset in Fig. 4). It is noted that, a small variation in $\beta_{\mathrm{T}}$ of order 0.00002 reduces the delay time significantly as shown in Fig. (4).

\section{Summary}

We have examined the switching response of the OB device consisting of twolevel atomic medium placed in a ring cavity and interacting with a single mode cavity field supported by KNB reservoir in the mean field limit and high-Q cavity case. The switching times for different reservoirs, such as, normal vacuum (NV), thermal field (TF), and squeezed vacuum (SV) are compared.

Two types of switching response are examined, namely:

(a) Field switching process, at fixed temperature of the KNB reservoir, shows that the delay time of the $\mathrm{OB}$ device is less than that of other OB systems with $\mathrm{NV}, \mathrm{TF}$ and $\mathrm{SV}$ cases. In the dispersive case, the increase in atomic detuning leads to an increase in delay time.

(b) Thermal switching process, at fixed input field, where a very slight change in the temperature of the device induces a switching with less delay. This behavior may be useful in proposing switch that is analogous to a conventional thermostat switch device in which electric current levels are 
controlled in such devices. In the suggested switch the light levels in the optical systems are controlled based on the change of temperature of the KNB reservoir. Recently, thermally-induced OB has been reported in silicone based-photonic crystal cavities due to the change of the refractive index through thermo-optic effect [37].

\section{References}

[1] H. M. Gibbs, Optical Bistability: Controlling Light with Light, Quantum electronics-principles and applications, Academic Press, Orlando, FL, 1985.

[2] A. Joshi and M. Xiao, Controlling Steady-state and Dynamical Properties of Atomic Optical Bistability, World Scientific, Singapore, (2012).

[3] R. Bonifacio and L. A. Lugiato, Cooperative effects and bistability for resonance fluorescence,Opt. Commun. $\underline{\mathbf{1 9}}$, (1976) 172-176.

[4] S. S. Hassan, P. D. Drummand and D. F. Walls, Dispersive optical bistability in a ring cavity, Opt. Commun. 27 (1978) 480-484.

[5] H. M. Gibbs, S. L. McCall and T. N. C. Venkatesan, Differential Gain and Bistability Using a Sodium-Filled Fabry-Perot Interferometer, Phys. Rev. Lett. $\underline{36}$ (1976) 1135-1138.

[6] E. Abraham and S. D. Smith, Optical bistability and related devices, Rep. Prog. Phys. $\underline{\mathbf{4 5}}$ (1982) 815-885.

[7] W. Harshawardhan and G. S. Agrawal, Controlling optical bistability using electromagnetic-field-induced transparency and quantum interferences ,Phys. Rev. A $\underline{\mathbf{5 3}}$ (1996) 1812-1817.

[8] S. Gong, S., Du, Z, Xu, S. Pan, Optical bistability via a phase fluctuation effect of the control field, Phys. Lett. A $\underline{\mathbf{2 2 2}}$ (1996) 237-240. 
[9] Y. A. Sharaby, A. Joshi, and S. S. Hassan, Optical Bistability beyond Rotating Wave Approximation Phys. Lett. A $\underline{374}$ (2010) 2188-2194.

[10] S. S. Hassan, Y. A. Sharaby, M. F. Ali and A. Joshi, Optical bistabilities of higher harmonics: Inhomogeneous and transverse effects, Phys. Lett. A $\underline{\mathbf{3 7 6}}$ (2010) 3555-3561.

[11] C.W. Gardiner, Inhibition of Atomic Phase Decays by Squeezed Light: A Direct Effect of Squeezing, Phys. Rev. Lett. $\underline{\mathbf{6}}$ (1986) 1917-1920.

[12] Z. Cheng, Squeezed thermal radiation state in a Kerr-nonlinear black

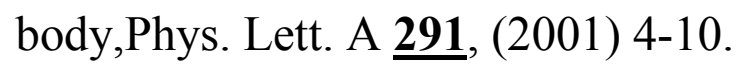

[13] M. Yin and Z. Cheng, Inhibited spontaneous emission in a Kerr nonlinear blackbod, Phys. Rev. A $\underline{\mathbf{7 8}}$, (2008) 0638290-0638297.

[14] P. Galatola, L. A. Lugiato, M. G. Porreca, P. Tombesi, Optical switching by variation of the squeezing phase, Opt. Commun. $\underline{\mathbf{8 1}}$ (1991) 175-178.

[15] S. S. Hassan, H. A. Batrafi, R. Saunders and R. K. Bullough, Bistable behaviour in squeezed vacua: I. Stationary analysis, Eur. Phys. J. D $\underline{8}$ (2000) 403416.

[16] H. A. Batrafi, S. S. Hassan, R. Saunders and R. K. Bullough, Bistable behaviour in squeezed vacua: II. Stability analysis and chaos, Eur. Phys. J. D $\underline{8}$ (2000) 417-429.

[17] S. M. A. Maize, M. F. M. Ali and S. S. Hassan, Squeezed vacuum effects on optical bistability in a Fabry-Perot cavity, Nonlinear Optics $\underline{8}$ (1994) 218-230. [18] J. Bergou and D. Zhao, Effect of a squeezed vacuum input on optical bistability, Phys. Rev. A $\underline{\mathbf{5 2}}$ (1995) 1550-1560.

[19] M. F. M. Ali, S. S. Hassan and S. M. A. Maize, Transverse field, squeezed vacuum and inhomogeneous broadening effects on optical bistability, J. Opt. B: Quantum Semiclass. Opt. 4 (2002) 388-395. 
[20] A. Joshi, Y. A. Sharaby and S. S. Hassan, Temperature-induced optical bistability with Kerr-nonlinear blackbody reservoir, Optics Commun. 359 (2016) 387-392.

[21] Q. F. Xu, X. Z.-Hui, J. N. Chen and Z. Cheng, Entanglement properties of atoms in the Kerr-nonlinear Blackbody, Eur. Phys. J. D $\underline{66}$ (2012) 66:86.

[22] Z. Cheng, Spectral energy density and radiation pressure in a Kerr nonlinear blackbody, Phys. Lett. A $\underline{\text { 331, }}$ (2004) 170-174.

[23] R. Bonifacio and P. Mystre, Critical slowing down in optical bistability ,Optics Comm. $\underline{29}$ (1979) 131-134.

[24] S. S. Hassan and Y. A. Sharaby, Critical slowing down in a bistable model with squeezed vacuum environment, Eur. Phys. J. D $\underline{\text { 30 }}$ (2004) 393-401.

[25] H. A. Al-Attar and H. A. MacKenzie and W. J. Firth,Critical slowing-down phenomena in an InSb optically bistable etalon, J. Opt. Soc. Am. B $\underline{\mathbf{3}}$ (1986) 1157-1163.

[26] S. Cribier, E. Giacobino and G. Grynberg, Quantitative investigation of critical slowing down in all-optical bistability, Optics Comm. 47 (1983) 170-172. [27] E. Garmire, J. H. Marburger, S. D. Allen ans H. G. Winful, Transient response of hybrid bistable optical devices, Appl.Phys. Lett. $\underline{\mathbf{3 4}}$ (1979) 374-376. [28] P. Mandel and T. Erneux, Nonlinear control in optical bistability, IEEE J. Quantum Electron. QE 21 (1985) 1352-1355.

[29] Y. A. Sharaby, S. S. Hassan and A. Joshi, Critical slowing down with bistable higher harmonics, Optics Comm. $\underline{311}$ (2013) 389-396.

[30] S. S. Hassan, Y. A. Sharaby, M. F. M. Ali and F. N. M. Al-showaikh, Inhomogeneous and transverse effects on critical slowing down with bistable higher harmonics, Nonlinear Optics, Quantum Optics 뚜, (2014) 35-50.

[31] R. A. Alharbey, L. A. M. Nejad, S. Lynch and S. S. Hassan, Critical slowing down in biological bistable models, Int. J. Pure \& Appl. Maths $\underline{93}$ (2014) 581602. 
[32] N. Wolchover, Nature's critical warning system, Quanta Magazine: https:/quantamagazine.org/20151117-natures-critical-warning-system/ (last accessed 04/06/16.

[33] Q. Shu and S.C. Rand, Critical slowing down and dispersion of avalanche upconversion dynamics, Phys. Rev. B 55 (1997) 8776-8783.

[34] H.A. Al-Attar, H.A. MacKenzie and W.J. Firth, Critical slowing down phenomena in an InSb optically bistable étalon, J. of Optical Society of America B 3, (1986) 1157-1163.

[35] Z. Cheng, Quantum effects of thermal radiation in a Kerr nonlinear blackbody, J. Opt. Soc. Am. B 19, (2002) 1692-1705.

[36] S. Lynch, Dynamical Systems with Applications using Mathematica, Springer (2007).

[37] R. Shankar, I. Bulu, R. Leijssen, M. Loncar, Study of thermally-induced optical bistability and the role of surface treatments in Si-based mid-infrared photonic crystal cavities, Opt. Exp. 19 (2011) 24828-24837.

\section{Figure captions}

Fig. 1: The transient output field component $|x(\tau)|$, versus the normalized time $\tau=\kappa \mathrm{t}$ for $\mathrm{C}=20, \delta=\theta=0, \beta=0.04$ and different bistable systems: $\mathrm{SV}(\longrightarrow)($ $\left.n_{S V}=0.158237, \Phi=\pi\right), \mathrm{NV}(\cdots \cdots)\left(\mathrm{n}=0, \Gamma_{r}=1\right), \operatorname{KNB}(-\cdot-\cdot)\left(n_{K N B}=0.158237\right.$, $\left.\Gamma_{r}=1 / 12\right), \operatorname{TF}(---)\left(n_{T F}=0.158237, \Gamma_{r}=1\right)$.

Fig. 2: The transient output field component $|x(\tau)|$, in the KNB case ( $\left.n_{K N B}=0.158237, \Gamma_{r}=1 / 12\right)$ case versus the normalized time $\tau=\kappa \mathrm{t}$ for $\mathrm{C}=20$, $\delta=\theta=0$, and different values of the input field perturbation; $\beta=0.0005(-)$, $\beta=.001(\cdots \cdots)$ and $\beta=0.008(---)$.

Fig. 3: The transient output field component $|x(\tau)|$, in the KNB case ( $\left.n_{K N B}=0.158237, \Gamma_{r}=1 / 12\right)$ versus the normalized time $\tau=\kappa \mathrm{t}$ for $\mathrm{C}=20, \theta=0$, 
$\beta=0.04$, and different values of the atomic detuning $\delta=0(-), \delta=1.5(\cdots \cdots)$ and $\delta=3(---)$.

Fig. 4: The transient output field component $|x(\tau)|$ versus the normalized time $\tau=\kappa \mathrm{t}$ in the high-Q limit for $\mathrm{C}=40, \theta=\delta=\Gamma_{\mathrm{r}}=1 / 2, \mathrm{Y}=80$ and different values of the relative temperature perturbation $T_{b}=T_{b C}+\beta_{T}, T_{b C}=0.543 ; \beta_{T}=0.00116$ $(\longrightarrow), \beta_{T}=0.00118(\cdots \cdots)$ and $\beta_{T}=0.004(---)$. Inset shows the contour bistable curve $\left(|x|\right.$ vs. $\left.T_{b}\right)$ at fixed $\mathrm{Y}=80$. 


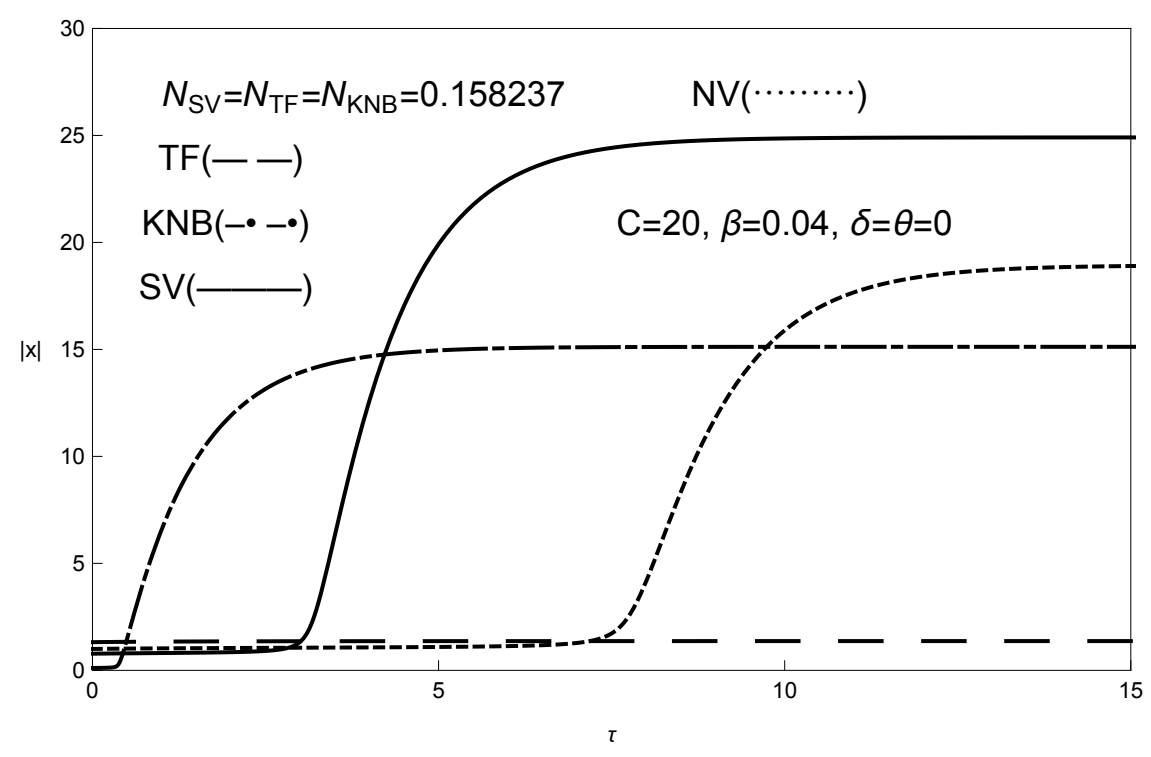

Fig. 1

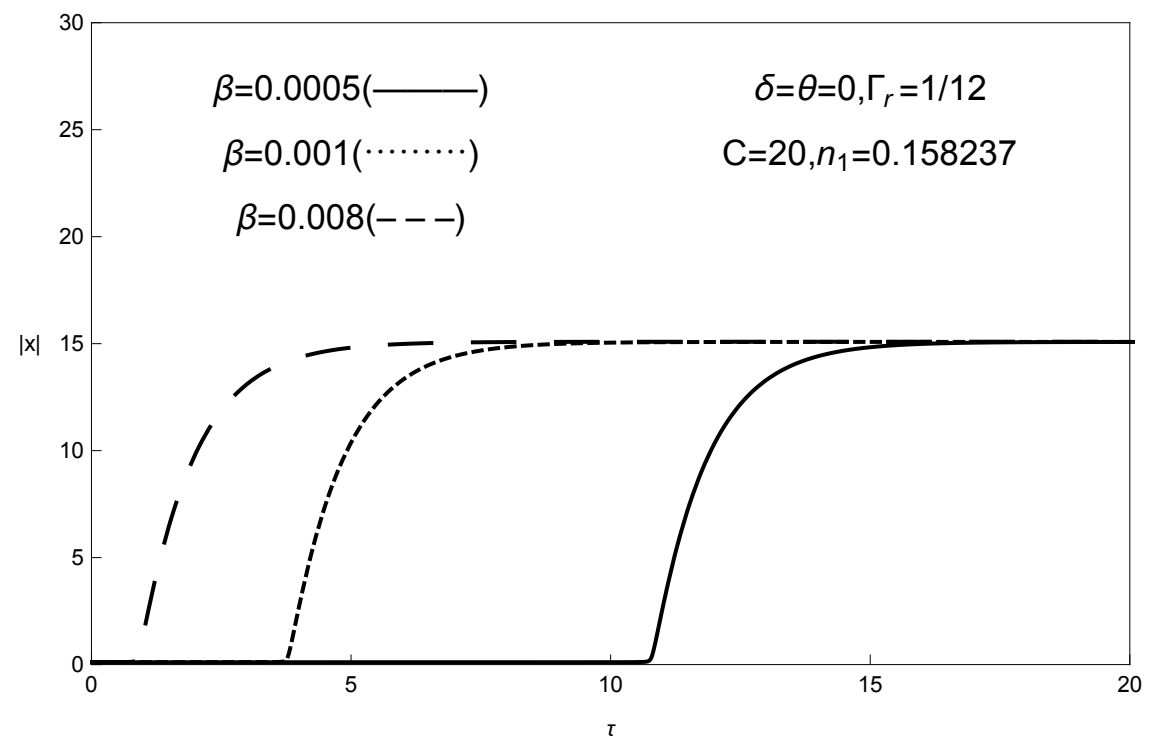

Fig. 2 


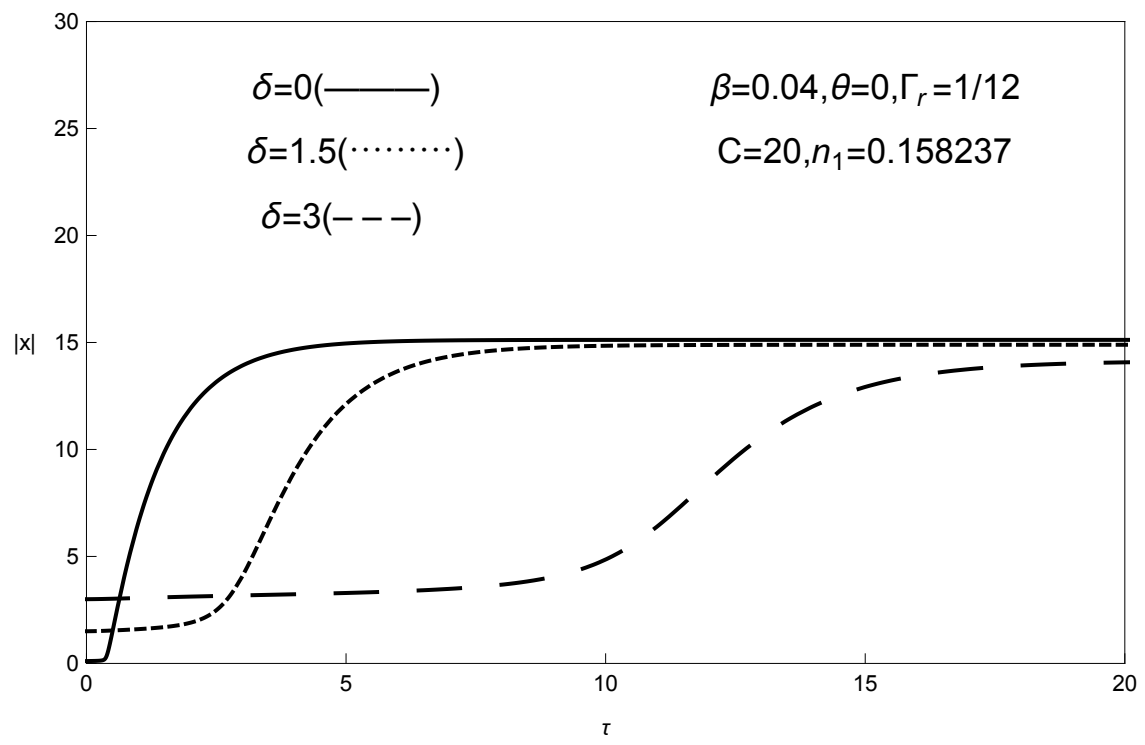

Fig. 3

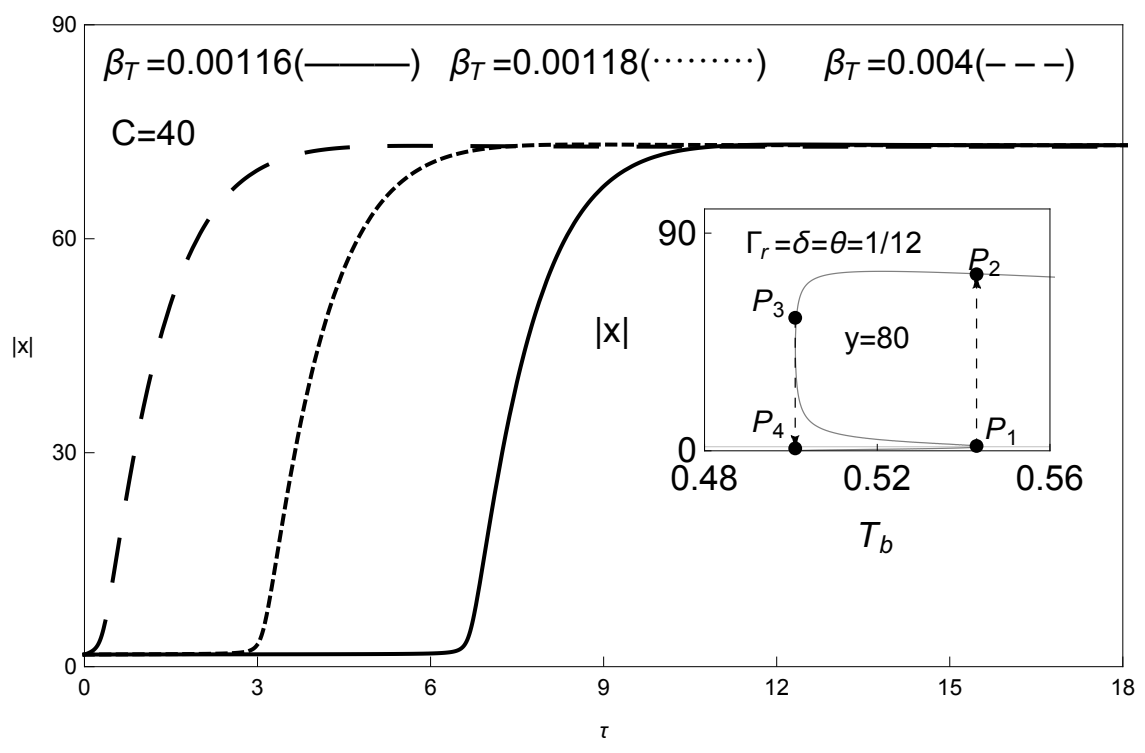

Fig. 4 